\title{
Real-Time Probabilistic Tracking of Faces in Video
}

\author{
Giuseppe Boccignone, Paola Campadelli, Alessandro Ferrari, and Giuseppe Lipori \\ Dipartimento di Scienze dell'Informazione - Università degli Studi di Milano \\ via Comelico 39/41, 20135 Milano, Italy \\ \{boccignone, campadelli, lipori\}@dsi.unimi.it, \\ alessandro.ferrari3astudenti.unimi.it
}

\begin{abstract}
In this note it is discussed how real-time face detection and tracking in video can be achieved by relying on a Bayesian approach realized in a multithreaded architecture. To this end we propose a probabilistic interpretation of the output provided by a cascade of AdaBoost classifiers. Results show that such integrated approach is appealing with respect either to robustness and computational efficiency.
\end{abstract}

\section{Introduction}

Face detection and tracking can be performed either according to a frame based approach (e.g., [1]) or according to a detection and tracking approach, where faces are detected in the first frame and tracked through the video sequence (for a review, refer to [2]). Clearly, in the first case, temporal information is not exploited, and the intrinsic independence among successive detections makes it difficult to reconstruct the track of each subject. In the second case a loss of information may occur (e.g., new faces entering the scene) and, in general, the output of face detection is used only at initialization, while tracking relies upon low level features (color histograms [3], contours [4], etc.) which are very sensitive to the conditions of acquisition. To overcome these drawbacks, a tighter coupling between face detection and tracking has been proposed [2]. Such an approach can be given a simple and elegant form in the Bayesian framework.

Each face is characterized at frame $t$ of the video stream by a state vector $\mathbf{x}_{t}$, e.g., a face bounding box. The tracking goal is to estimate the correct state $\mathbf{x}_{t}$ given all the measurements $Z_{t}=\left\{z_{1}, \cdots, z_{t}\right\}$ up to that moment, or equivalently to construct the posterior probability density function (pdf) $p\left(\mathbf{x}_{t} \mid Z_{t}\right)$. The theoretically optimal solution is provided by recursive Bayesian filtering that in the prediction step uses the dynamic equation and the already computed pdf of the state at time $t-1, p\left(\mathbf{x}_{t-1} \mid Z_{t-1}\right)$, to derive the prior pdf of the current state, $p\left(\mathbf{x}_{t} \mid Z_{t-1}\right)$; then, in the update step, it employs the face likelihood function $p\left(z_{t} \mid x_{t}\right)$ of the current measurement to compute the posterior pdf $p\left(\mathbf{x}_{t} \mid z_{t}\right)$. Formally:

$$
p\left(\mathbf{x}_{t} \mid Z_{t}\right) \propto p\left(z_{t} \mid \mathbf{x}_{t}\right) \int p\left(\mathbf{x}_{t} \mid \mathbf{x}_{t-1}\right) p\left(\mathbf{x}_{t-1} \mid Z_{t-1}\right) \mathrm{d} \mathbf{x}_{t-1} .
$$

Thus, the key issue of a tight coupling approach is to provide a face detection algorithm suitable to calculate the face likelihood function $p\left(z_{t} \mid \mathbf{x}_{t}\right)$ and, if a Particle Filtering 
(PF, [4]) implementation of Eq. 1 is adopted, to generate proper particle weighting. For instance, Verma et al. [2] adopt the Schneiderman and Kanade detector [5], which provides a suitable probabilistic output, but unfortunately is inadequate for real-time implementation.

A more appealing solution, which we propose here, could be the real-time face detection scheme proposed by Viola and Jones (VJ, [6]) - basically a cascade of AdaBoost classifiers - which is arguably the most commonly employed detection method [7]. Note that the combination of AdaBoost and PF has been proposed in [3], but detection results were heuristically combined in the proposal function, rather than being exploited to model a likelihood function in a principled way. In [8], PF is integrated with an AdaBoost monolithic classifier via the probabilistic interpretation given by Friedman et al. in [9]. Unfortunately tight coupling is spoiled by the introduction of a mean shift iteration, based on color features, to support the prediction of new hypotheses in adjacent regions.

Differently, we exploit the cascaded classifiers described in [10]. In this case, the probabilistic interpretation valid for monolithic classifiers does not apply directly; thus we work out a probabilistic interpretation of the VJ algorithm suitable to address Eq. 1 (Section 2).

Section 3 discusses the proposed PF implementation of Eq. 1 by defining the state parametrization and the adopted dynamical model. A PF may perform poorly when the posterior is multi-modal as the result of ambiguities or multiple targets, and issues such as appearance and disappearance of faces should be handled in a robust way. Interestingly enough, the latter issue, which is considered critical in PF based tracking, is very easily solved in human vision where multiple object tracking "runs" in parallel with background motion alerting processes capable of triggering, through pop-out effects, the tracking of new objects entering the scene and the loss of attention for disappearing objects. These problems have been tackled by incorporating such concurrency of processes in the system architecture. Experimental results and performance of the system are discussed in Section 4

\section{Face Likelihood: A Probabilistic View of Viola and Jones}

AdaBoost classifiers are originally conceived for binary classification, attributing to an example $\mathbf{x}$ a label $y \in\{-1,1\}$ according to the sign of the weighted $\operatorname{sum} F(\mathbf{x})=$ $\sum_{i} w_{i} \cdot h_{i}(\mathbf{x})$, where $h_{i}$ are the so called weak classifiers and $w_{i}$ the associated weights. In our case a typical example is specified by a state vector $\mathbf{x}=\{x, y$, width, height $\}$ that indicates the location and size (in pixels) of a rectangular box surrounding the face. According to Friedman et al. [9], the following holds:

$$
p(y=1 \mid \mathbf{x})=p(y=1 \mid F(\mathbf{x}))=\frac{e^{F(\mathbf{x})}}{e^{F(\mathbf{x})}+e^{-F(\mathbf{x})}} .
$$

In our interpretation, $p(z \mid \mathbf{x}) \simeq p(y=1 \mid \mathbf{x})$ since the application of the face classifier is the means by which data are observed. Hence, for monolithic classifiers, Eq. 2 can be directly plugged in Eq. 1. Unfortunately, in case of a cascaded classifier, the likelihood estimate is not as immediate. 
A cascaded classifier is obtained by chaining a set of monolithic classifiers or stages so to increase the specialization of the classifiers along the cascade. An example is classified as a positive if and only if it is judged so by all stages, while negative examples are discarded according to an early reject strategy. Along training, each stage falsely accepts a fixed ratio $f$ of the non-face patterns in the training sample, while wrongly eliminating only a very small portion $1-d$ of face patterns; formally:

$$
\begin{aligned}
& p\left(F_{i}(\mathbf{x}) \geq 0 \mid F_{i-1}(\mathbf{x}) \geq 0, y=1\right)=d \\
& p\left(F_{i}(\mathbf{x}) \geq 0 \mid F_{i-1}(\mathbf{x}) \geq 0, y=-1\right)=f
\end{aligned}
$$

where $F_{i}(\mathbf{x})$ is the weighted sum output by the $i$-th stage classifier, $i=1, \ldots, k$, and $k$ is the total number of stages. Denote $F_{i}^{+}$the event $F_{i}(\mathbf{x}) \geq 0$ of positive classification at stage $i$ and $F_{i}^{-}$the complementary event.

By applying the conditional rule of probability and recursively exploiting the Markovianity intrinsic to the cascade, the global detection and false acceptance rates of the trained cascade are easily derived as

$$
\begin{aligned}
& d_{g}=p\left(F_{k}^{+}, \ldots, F_{1}^{+} \mid y=1\right)=d^{k} \\
& f_{g}=p\left(F_{k}^{+}, \ldots, F_{1}^{+} \mid y=-1\right)=f^{k} .
\end{aligned}
$$

If the training examples are representative of the learning task, one could expect similar detection and false alarm rates also when applying the cascade to test examples.

We are interested in evaluating the likelihood of an example under the two possible outcomes of classification by the cascade, namely $p\left(y=1 \mid F_{k}^{+}, \ldots, F_{1}^{+}\right)$and $p\left(y=1 \mid F_{i}^{-}, F_{i-1}^{+}, \ldots, F_{1}^{+}\right), i=1, \ldots, k$. By applying Bayes' rule, the conditional rule of probability, Markovianity and Eqs. 5 and 6 .

$$
\begin{aligned}
p\left(y=1 \mid F_{k}^{+}, \ldots, F_{1}^{+}\right) & =\frac{p\left(F_{k}^{+}, \ldots, F_{1}^{+} \mid y=1\right) p(y=1)}{\sum_{\bar{y} \in\{-1,1\}} p\left(F_{k}^{+}, \ldots, F_{1}^{+} \mid y=\bar{y}\right) p(y=\bar{y})} \\
& =\frac{d^{k} p(y=1)}{d^{k} p(y=1)+f^{k} p(y=-1)} \\
p\left(y=1 \mid F_{i}^{-}, F_{i-1}^{+}, \ldots, F_{1}^{+}\right) & =\frac{p\left(F_{i}^{-}, F_{i-1}^{+} \ldots, F_{1}^{+} \mid y=1\right) p(y=1)}{\sum_{\bar{y} \in\{-1,1\}} p\left(F_{i}^{-}, F_{i-1}^{+}, \ldots, F_{1}^{+} \mid y=\bar{y}\right) p(y=\bar{y})} \\
& =\frac{(1-d) d^{i-1} p(y=1)}{(1-d) d^{i-1} p(y=1)+(1-f) f^{i-1} p(y=-1)}(8)
\end{aligned}
$$

The only unknown left is $p(y=1)$, i.e. the probability of feeding the cascade with a true positive example; assuming once again that the cascade is applied to examples drawn according to the same distribution that generated the training set, one can force this value to the ratio between the cardinalities of the positive and negative classes in the training set.

It is worth spending some words about the assumption according to which the distributions over the training and test set are taken to be the same. On one side it is clear that 
this hypothesis does not hold strictly: it is sufficient to notice that, due to the nature of particle filtering, during tests many particles will be placed in the neighborhood of the face although not perfectly aligned with the pattern. Since the original training sample contains only perfectly aligned face regions, most of such particles are likely not to be classified as positives by a monolithic classifier. On the other hand, one can expect these examples to pass through a bigger number of stages (loosely inversely proportional to the degree of misplacement) with respect to true negative patterns, resulting in a higher likelihood and hence contributing to the correct estimation of the density distribution.

Moreover, the same issue applies also to particles generated under other circumstances which are not taken into account by the original training, such as out-of-plane rotations. One possible approach is that of [8], where out-of-plane rotations are embedded in the training set to extend the class of positives. In this spirit misalignment should be also included; we think that this approach would introduce too much variability for a learning technique which is based on Haar filter responses and is therefore highly dependent on the structure and relative arrangement of facial features.

In this work our interest is not that of fine tuning the face detector; instead, we intend to reuse the off-the-shelf detector described in [10] as a plug-in module into a PF scheme 1 . Rotations around the vertical axis are taken into account by means of combining the profile classifier; other out-of-plane rotations are considered in the same way as sequences of occlusions and treated as discussed in Section 4

\section{Face Tracking}

Face tracking is accomplished via Particle Filtering [4]. The main idea of PF relies upon approximating the probability distribution by means of a set of weighted samples $S=\left\{\left(\mathbf{s}^{(n)}, \pi^{(n)}\right)\right\}, n=1, \ldots, N$. A sample $\mathbf{s}$ represents a hypothetical state of the tracked object to which is associated a discrete sampled probability $\pi$, where $\sum_{n=1}^{N} \pi^{(n)}=1$. The goal is to compute by sampling the posterior probability $p\left(\mathbf{s}_{t} \mid Z_{t}\right)$ in place of $p\left(\mathbf{x}_{t} \mid Z_{t}\right)$. That is, filtering is performed by rewriting Eq. 1 as $p\left(\mathbf{s}_{t} \mid Z_{t}\right) \propto p\left(z_{t} \mid \mathbf{s}_{t}\right) \int p\left(\mathbf{s}_{t} \mid \mathbf{s}_{t-1}\right) p\left(\mathbf{s}_{t-1} \mid Z_{t-1}\right) \mathrm{d} \mathbf{s}_{t-1}$.

In our case $\mathbf{s}_{t}=\{x, y, s, \theta\}$, where $s$ is the side of the square face bounding box and $\theta$ indicates the face pose (rotation around the vertical axis) estimated as in [2] by interpolating the likelihood of the particle as measured by the frontal and profile classifier.

A filtering cycle starts with resampling $S_{t-1}$ by extracting $N$ particles with probability $\pi_{t-1}^{(n)}$ to populate the new set $S_{t}$, as described in [4]. Then prediction $p\left(\mathbf{s}_{t} \mid \mathbf{s}_{t-1}\right)$ is obtained by diffusing each particle by a zero order dynamical model, with some adjustments w.r.t. [2]:

$$
\begin{array}{rlrl}
x_{t}=x_{t-1}+\mathcal{N}\left(0, \sigma_{x}^{2}\right) \cdot s_{t-1} \cdot \phi, & & y_{t}=y_{t-1}+\mathcal{N}\left(0, \sigma_{y}^{2}\right) \cdot s_{t-1} \cdot \phi \\
s_{t}=s_{t-1}(1.1)^{\left\lfloor 0.5+\mathcal{N}\left(0, \sigma_{s}^{2}\right) \cdot \phi\right\rfloor}, & \theta_{t}=\theta_{t-1}+\mathcal{N}\left(0, \sigma_{\theta}^{2}\right) \cdot \phi
\end{array}
$$

\footnotetext{
${ }^{1}$ Any other classifier among those available in the OpenCV distribution for full, lower or upper body detection could be used equally well.
} 
where $\sigma_{x}$ and $\sigma_{y}$ are percentage values to relate the diffusion to the particle size, and $\phi$ is a factor depending on the frame rate of acquisition. Subsequently, data observation is accomplished and the likelihood $p\left(z_{t} \mid \mathbf{s}_{t}\right)$ evaluated, as explained in Section 2. Eventually, the weighted mean state is computed as $\mu_{\mathbf{s}}=E[\mathbf{s}]=\sum_{n=1}^{N} \pi^{(n)} \mathbf{s}^{(n)}$, and this determines the estimated position of the face.

In principle, the original PF formulation [4] allows to track multi-target / multi-modal distributions. In practice, this requires to automatically identify and separate the different components (modes), ensuring that each has a sufficient number of particles to evolve correctly. To avoid this, we choose to keep the components separated by activating a different set of particles for every detected subject. To activate the PF cycle, initialization is triggered by a motion detection module [11], that executes as a background process (thread); the system stays in "stand-by" till a certain amount of motion is seen in the scene. In that case, the system runs a face detection scan over the regions which represent moving objects in order to locate the subjects, and then launches a new PF thread for each detected face. This mechanism allows to spare system resources by executing face detection only where and when needed, so avoiding periodic re-initialization as in [2] which would require exhaustive scans over the entire frame (all positions, all scales).

Once initialization is accomplished, all threads run in parallel. Similarly to [2], each sample of particles is constantly monitored in order to automatically detect when the face estimate looses track of the subject for some reason. In particular, a sufficient condition is whether the maximum likelihood over the sample is below a threshold $p_{\min }$ for more than $N_{1}$ of frames, or if the standard deviation over the sample of one of the state dimensions $\{x, y, s, \theta\}$ exceeds the corresponding threshold $\left\{x_{\max }, y_{\max }, s_{\max }, \theta_{\max }\right\}$ for more than $N_{2}$ of frames. In both cases, the PF thread tries to re-initialize the sample by performing face detection in a ROI including the recent movement of the subject; in case of failure for more than a certain number of trials, the thread exits and the sample dies out.

If desired, e.g. in situations where subjects can enter/exit only from outside the scene, the system can exploit a definition of peripheral vision to treat certain events such as the appearance of a new face in the scene, or the disappearance of a tracked face. The first situation takes place when the motion detector segments a blob of peripheral moving foreground with no intersection with the ROIs of existing trackers, therefore it is handled within the main thread by performing selective face detection on the blob to initialize a new PF thread. The second event involves a subject who is already being tracked, hence it is treated as a special case of track loss when the ROI including the recent movement of the subject terminated in the peripheral area of the frame. However, in more general scenarios where no assumption can be made about the entry/exit location of subjects (e.g. when monitoring a corridor), the notion of peripheral area can be disabled and all events are equally treated independently from their position, at the expenses of a minor increase in demand of computational resources.

The multi-threaded tracking system can run in two modalities: unsynchronized and synchronized. In the former, the main thread signals to the PF threads when a new frame is available from the streaming, but does not wait for them to complete execution of the filtering cycle. In case a PF thread loses one or more frames, it will adjust the prediction 
step by setting the diffusion parameter $\phi$ to the number of frames elapsed from the last cycle execution. In the synchronized case, the newly available frame is signaled only when all PFs have accomplished their task; this modality of execution can be simulated by a single-threaded system and will eventually slow down the global performance when several subjects are tracked simultaneously.

\section{Experimental Setting and Results}

Data set. We gathered two sets of short video sequences: 1) 16 validation sequences for tuning the main parameters 2) 5 test sequences to evaluate the global behavior of face tracking. The sequences have been shot in such a way to isolate the sources of variability, making the tuning of the different parameters as independent as possible. In particular, there are sequences of horizontal motion to tune $\sigma_{x}$, vertical motion for $\sigma_{y}$, motion in depth for $\sigma_{s}$, and sequences with face occlusions for $p_{\min }, x_{\max }, y_{\max }, s_{\max }$. For each sequence type we acquired 4 videos to enrich the sample variability, making a total of 16. Regarding each type of motion, we shot two sequences with uniform dynamics (one slower, one at normal speed), a sequence with speed discontinuities (pauses) and one with changes in direction. Regarding occlusions, the four sequences vary in the duration of the occlusion $(23,35,50$, and 80 frames), however never exceeding the parameters of tracking persistence (in our experiments $N_{1}=N_{2}=100$ ). The cardinality of each sample is set to $N=100$ particles.

Motion parameters. These were separately tuned in $[0.1,0.5]$ with unform increment of 0.05; the optimal values are those that minimize the number of frames in which the tracking mean estimate is not well positioned and scaled over the subject face, as judged by visual inspection. Doing so, we set $\sigma_{x}=0.15$ ( $0.3 \%$ of lost frames), $\sigma_{y}=0.15$ $(1 \%)$, and $\sigma_{s}=0.4(15.7 \%)$. As expected, the scale dynamics is the most difficult to correctly predict. In our experience, this is mainly due to an inferior sensitivity of the face detector with respect to the scale parameter, which is compensated, in our system, by the re-initialization mechanism so that in no tuning sequences the tracking was definitely lost. Although it is convenient to tune each motion parameter separately to reduce the number of experiments, the results cannot be applied as they are on sequences with mixed dynamics because the different components of diffusion increase the sample variability in a non linear way. Therefore, we consider a joined reduction of the estimated diffusion parameters by about $50 \%$, leading to the following configuration: $\sigma_{x}=0.1, \sigma_{y}=0.1$ and $\sigma_{s}=0.25$.

Pose estimation. In the vein of [2], we exploit the combined application of two specialized classifiers, one for frontal and one for profile views, in order to estimate the correct pose angle of the tracked subjects. To do so, we used the classifiers made available in the OpenCV library distribution [12] with no need to retrain. Notice that the standard deviation of the pose parameter $\sigma_{\theta}$ is not subject to tuning since it does not contribute to the observation likelihood, hence the system is almost unaffected by variations of $\sigma_{\theta}$ (a little diffusion is still necessary for correct inference of pose side, so in our experiments $\sigma_{\theta}=1^{\circ}$. 


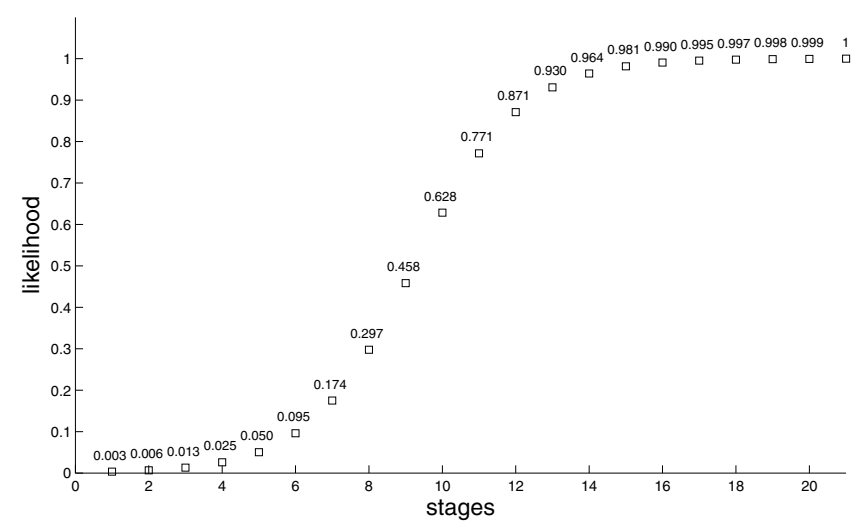

Fig. 1. The tabulated likelihood values in the event of rejection by the cascade (positions 1 to $k$ ) and in case of positive classification (position $k+1$ )

Observation likelihood computation. The method derived in Section 2 offers the advantage that the likelihood is estimated based only on training data, hence it can be pre-computed and stored in a look up table that contains at positions $i=1, \ldots, k$ the likelihood associated to examples rejected by the $i$-th stage of the cascade, and (for convenience) at position $k+1$ the likelihood of an example classified as a positive. Since we are using Lienhart's algorithms [10] as implemented in [12] we can straightforwardly refer to the training data reported in [10], namely $p(y=1)=\frac{5}{8}, d=0.999$, $f=0.5, k=20$ and hence $d_{g} \approx 0.98, f_{g} \approx 9.6 e-7$, yielding the weights in Fig. 1 These values refer to the training of the frontal classifier, while no mention is made to the corresponding values for training the profile classifier. For simplicity we assume it was trained over a sample with the same positive-to-negative ratio and with the same requirements $d$ and $f$ put on each stage.

Occlusions. We observed that during such an event all particles are attributed with equally low weights, which in turn results in an increasingly bigger dispersion of the sample. Following these considerations, we separately tuned the probability parameter, $p_{\min }$, from the standard deviation thresholds, $x_{\max }, y_{\max }, s_{\max }$. Regarding the former, the goal is to set the threshold in such a way that a tracker promptly reacts to occlusions of the face. To this end, we varied $p_{\min }$ among the likelihood values corresponding to the last 6 stages (Fig. 1) and chose the one that minimized the percentage of occluding frames not signaled as problematic, leading to $p_{\min }=0.999(0.5 \%$ of false detections). Regarding the standard deviations, we plot their values against time and chose the thresholds that best separated the intervals of occlusions from the other frames, leading to the following configuration: $x_{\max }=5, y_{\max }=5$ and $s_{\max }=3$.

Entry/exit events. There are no parameters directly involved in the strategy for entry/exit detection on/off the scene.

Results. After tuning, the method has been tested on 5 sequences of different types, for a total of 5327 frames. Results are measured by visual inspection and refer to the 

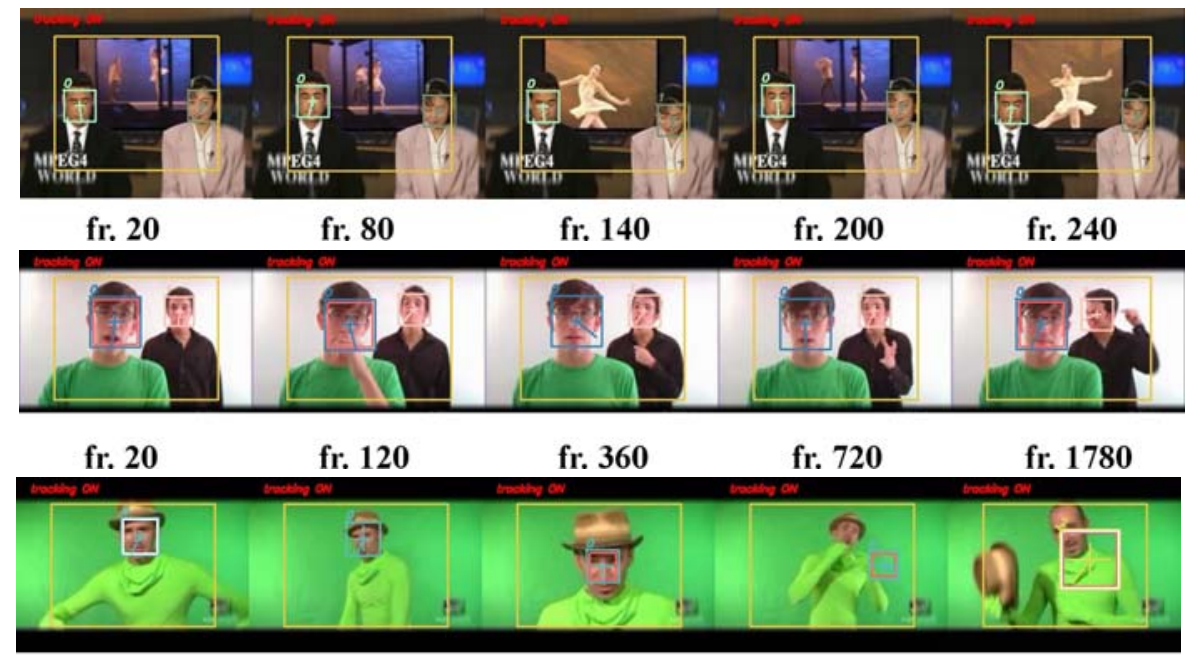

fr. 20

fr. 120

fr. 220

fr.320

fr. 720

Fig. 2. Excerpts from the news, dialogue and chroma key sequences. Yellow box encloses the central area of vision as opposed to the peripheral.

following events: percentage of frames with erroneous estimate of the face, erroneous changes of identity (failed re-initializations), number of tracked false positives (which usually expire after $\max \left(N_{1}, N_{2}\right)$ frames). Table 1 summarizes the obtained results and Figures 2 and 3 depict excerpts from the test sequences. The sequences cover various situations: face expression and verbalization (dialogue, news, chroma key), complex and moving background (video clip, news), entry in the scene (video clip, MAC vs PC), pose variations (video clip, MAC vs PC). The worst behavior was observed on the chroma key sequence, which is characterized by chaotic movements with sudden changes of direction, and quick motion in depth; the observed dynamics is far beyond the dynamics that can be modelled by our stochastic diffusion. Some problems were also observed on
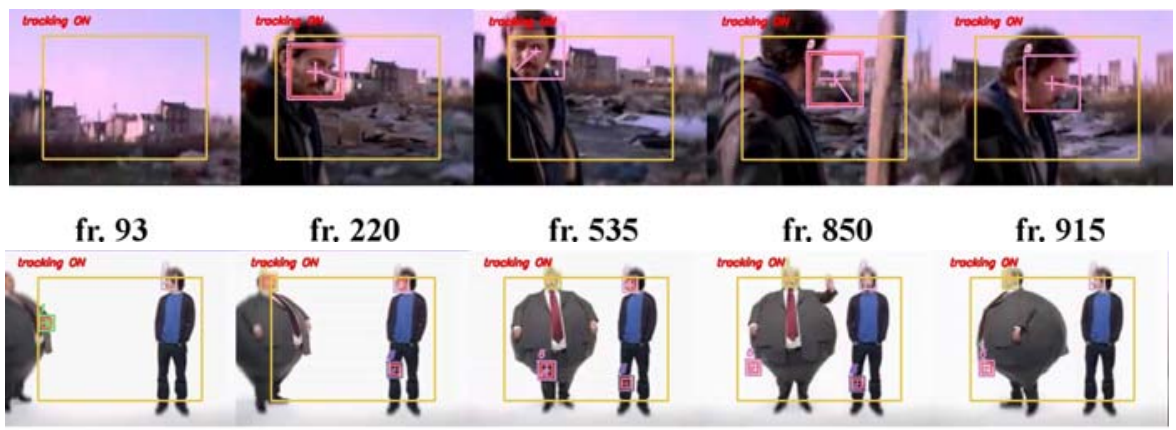
fr. 93
fr. 220
fr. 535
fr. 850
fr. 915

Fig. 3. Excerpts from the video clip and $M A C v s P C$ sequences 
Table 1. Results on test sequences

\begin{tabular}{|c|c|c|c|c|}
\hline sequence & frames & track loss & ID changes & false positives \\
\hline news & 298 & $0 \%$ & 0 & 0 \\
dialogue & 2495 & $0 \%$ & 0 & 0 \\
chroma key & 1022 & $70.1 \%$ & 2 & 1 \\
video clip & 921 & $14.4 \%$ & 0 & 0 \\
MAC vs PC & 636 & $2.8 \%$ & 0 & 4 \\
\hline
\end{tabular}

the video clip sequence, in occasion of the subject turning away from the camera (pose angle beyond $90^{\circ}$, see Fig. 3).

The system runs at about $15 \mathrm{fps}$ on videos of $320 \times 240$ pixels (minimal required resolution for faces is $20 \times 20$ pixels); the frame rate raises to 18 if the motion detection module is turned off (assuming no subjects are entering the scene), and to $64 \mathrm{fps}$ if the pose estimation is also disabled. These measurements have been taken on a Pentium D 3.4GHz CPU, under MS Windows XP operating system. Current software implementation is in $\mathrm{C}++$ language.

\section{Conclusions}

Main results achieved so far can be summarized as follows: 1) a principled integration of face detection and tracking steps, relying on a novel probabilistic interpretation of the $\mathrm{VJ}$ detection algorithm; 2) this turns into an efficient implementation of the likelihood computation in terms of look-up table; 3 ) introduction of a multi-threaded architecture which contributes to effective handling of event detections within the scene.

Preliminary results show that the proposed approach is appealing with respect to either robustness and computational efficiency. Concerning accuracy, that is the precision of the face estimate regarding position, scale and pose, it depends on the number of particles constituting every tracking sample, and especially on the virtues of the particular face detection technique employed in the PF scheme. It is generally believed that the Viola and Jones detector is a very efficient technique, but not as accurate; in fact, we observe that the tracking estimate is not always precise and cannot be used as it is for initializing further processing techniques of facial features.

A first improvement in that sense could be obtained by applying some temporal smoothing over the estimate. Moreover, the accuracy would also benefit from the introduction of a deterministic component in the prediction step to better condition the evolution; this component could be estimated online during execution, or even learned in case of very specific tracking tasks. Finally, one could substitute the OpenCV detector with any other cascaded Adaboost classifier that proved to be more accurate, if available, and plug it in directly into the framework by computing the associated likelihood as proposed in this article.

\section{Acknowledgements}

The authors acknowledge partial support by the PASCAL2 Network of Excellence under EC grant no. 216886. This publication only reflects the authors' views. 


\section{References}

1. Boccignone, G., Marcelli, A., Napoletano, P., Di Fiore, G., Iacovoni, G., Morsa, S.: Bayesian integration of face and low-level cues for foveated video coding. IEEE Trans. Circ. Sys. Video Tech. 18, 1727-1740 (2008)

2. Verma, R., Schmid, C., Mikolajczyk, K.: Face Detection and Tracking in a Video by Propagating Detection Probabilities. IEEE Transactions on Pattern Analysis and Machine Intelligence 25(10), 1215-1228 (2003)

3. Okuma, K., Taleghani, A., de Freitas, N., Little, J., Lowe, D.: A Boosted Particle Filter: Multitarget Detection and Tracking. LNCS, pp. 28-39. Springer, Heidelberg (2004)

4. Isard, M., Blake, A.: CONDENSATION - Conditional Density Propagation for Visual Tracking. International Journal of Computer Vision 29(1), 5-28 (1998)

5. Schneiderman, H., Kanade, T.: A statistical method for 3D object detection applied to faces and cars. In: Proc. IEEE Conf. Computer Vision and Pattern Recognition (2000)

6. Viola, P., Jones, M.: Robust Real-Time Face Detection. International Journal of Computer Vision 57(2), 137-154 (2004)

7. Pantic, M., Pentland, A., Nijholt, A., Huang, T.: Human Computing and Machine Understanding of Human Behavior: A Survey. In: Huang, T.S., Nijholt, A., Pantic, M., Pentland, A. (eds.) ICMI/IJCAI Workshops 2007. LNCS (LNAI), vol. 4451, pp. 47-71. Springer, Heidelberg (2007)

8. Li, P., Wang, H.: Probabilistic Object Tracking Based on Machine Learning and Importance Sampling. In: Marques, J.S., Pérez de la Blanca, N., Pina, P. (eds.) IbPRIA 2005. LNCS, vol. 3522, pp. 161-167. Springer, Heidelberg (2005)

9. Friedman, J., Hastie, T., Tibshirani, R.: Additive logistic regression: a statistical view of boosting. Technical report, Stanford University (1998)

10. Lienhart, R., Kuranov, E., Pisarevsky, V.: Empirical analysis of detection cascades of boosted classifiers for rapid object detection. In: DAGM 25th Pattern Recognition Symposium (2003)

11. Elgammal, A., Duraiswami, R., Harwood, D., Davis, L.S.: Background and foreground modeling using nonparametric kernel density estimation for visual surveillance. Proceedings of the IEEE 90(2) (2002)

12. OpenCV library: http://sourceforge.net/projects/opencvlibrary/ 